JHU-TIPAC-920022

MPI-Ph/92-84

October 1992

\title{
Harmonic potentials for quaternionic symmetric $\sigma$-models
}

\author{
A. Galperin ${ }^{(a) \dagger}$ and O. Ogievetsky ${ }^{(b) \ddagger}$ \\ (a) Department of Physics and Astronomy \\ Johns Hopkins University, Baltimore, MD 21218, USA \\ (b) Max-Planck-Institut für Physik, Werner-Heisenberg-Institut \\ Föhringer Ring 6, 8000 Munich 40, West Germany
}

\begin{abstract}
We construct $N=2$ superspace Lagrangians for quaternionic symmetric $\sigma$ models $G / H \times S p(1)$, or equivalently, quaternionic potentials for these symmetric spaces. They are homogeneous $H$ invariant polynomials of order 4 which are similar to the quadratic Casimir operator of $H$. The construction is based on an identity for the structure constants specific for quaternionic symmetric spaces.
\end{abstract}

$\dagger$ On leave from the Laboratory of Theoretical Physics, JINR, Dubna, Russia

$\ddagger$ On leave from P.N. Lebedev Physical Institute, Theoretical Department, 117924 Moscow, Leninsky prospect 53, Russia 


\section{Introduction}

Quaternionic spaces naturally appear in physics as solutions to self-dual gravity with a cosmological constant [1] as well as target spaces of $\sigma$-models in $N=2$ matter couplings to $N=2$ supergravity [2]. A general description of these $\sigma$-models in $N=2$ superspace was developed in [3]. It is based on the notion of harmonic superspace [4]; for a detailed exposition of this approach to quaternionic manifolds see [5]. In this description all the information about the quaternionic metric on the target space is contained in the quaternionic potential $\mathcal{L}^{+4}$. The latter is an analog of the Kähler potential for Kähler manifolds relevant to $N=1$ supersymmetry [6].

The $N=2$ superspace action for the general $4 n$-dimensional quaternionic $\sigma$-model has the following form

$$
S=\int d \zeta^{-4} d u\left\{Q_{\alpha}^{+} D^{++} Q^{+\alpha}-q_{i}^{+} D^{++} q^{+i}+\mathcal{L}^{+4}\left(Q^{+}, q^{+}, u^{-}\right)\right\} .
$$

Here integration is performed over the $N=2$ analytic superspace with coordinates $\zeta, u_{i}^{ \pm}$, $Q_{\alpha}^{+}(\zeta, u), \alpha=1, \ldots, 2 n$ and $q_{i}^{+}(\zeta, u), i=1,2$ are analytic $N=2$ superfields, $u_{i}^{ \pm}, i=1,2$ are the $S U(2) / U(1)$ isospinor harmonics, $u^{+i} u_{i}^{-}=1$. Finally, $D^{++}$is a supercovariant derivative with respect to harmonics. Its only property we will need is $D^{++} u_{i}^{-}=u_{i}^{+}$(for details and notation see [4], [3]).

The quaternionic potential $\mathcal{L}^{+4}$ is homogeneous in $Q^{+}, q^{+}$of degree two, it carries $U(1)$-charge +4 , does not depend on $u^{+}$and otherwise is an arbitrary real function. The reality is defined by the ordinary complex conjugation combined with the involution $u_{i}^{+} \mapsto u_{i}^{-}, u_{i}^{-} \mapsto-u_{i}^{+}$. The action (1) bears a remarkable analogy to the Hamiltonian mechanics [7] with the harmonic derivative $D^{++}$similar to time derivative, $Q^{+}, q^{+}$to phase space coordinates and the Poisson brackets given by

$$
\{f, g\}^{--}=\frac{1}{2} \Omega^{\alpha \beta} \frac{\partial f}{\partial Q^{+\alpha}} \frac{\partial g}{\partial Q^{+\beta}}-\frac{1}{2} \epsilon^{i j} \frac{\partial f}{\partial q^{+i}} \frac{\partial g}{\partial q^{+j}}
$$

where $\Omega^{\alpha \beta}$ and $\epsilon^{i j}$ are the invariant antisymmetric $S p(n)$ and $S p(1)$ tensors, respectively, and $Q^{+\alpha}=\Omega^{\alpha \beta} Q_{\beta}^{+}, q^{+i}=\epsilon^{i j} q_{j}^{+}$. The quaternionic potential $\mathcal{L}^{+4}$ is quite analogous to the Hamiltonian in mechanics; we will call $\mathcal{L}^{+4}$ Hamiltonian, too.

Isometries of the $\sigma$-model (1) are generated by Killing potentials $K_{A}^{++}\left(Q^{+}, q^{+}, u^{-}\right)$ which should obey the conservation law

$$
\partial^{++} K_{A}^{++}+\left\{K_{A}^{++}, \mathcal{L}^{+4}\right\}^{--}=0
$$

Under the Poisson brackets (2), the Killing potentials form the Lie algebra of the isometry group,

$$
\left\{K_{A}^{++}, K_{B}^{++}\right\}^{--}=f_{A B}{ }^{C} K_{C}^{++} \text {. }
$$

Here $\partial_{++}$is a partial derivative with respect to harmonics, $\partial_{++} u_{i}^{-}=u_{i}^{+}$.

An interesting problem is to identify Hamiltonians $\mathcal{L}^{+4}$ for known quaternionic manifolds. The simplest among the latter are quaternionic symmetric spaces described in 8 . There is precisely one compact and one noncompact quaternionic cosets for each simple 
complex Lie group. In $N=2$ supergravity one encounters the noncompact versions of these:

$$
\begin{array}{ccccc}
\frac{S U(n, 2)}{U(n) \times S p(1)} & \frac{S O(n, 4)}{S O(n) \times S U(2) \times S p(1)} & \frac{S p(n, 1)}{S p(n) \times S p(1)} & \frac{G_{2(+2)}}{S U(2) \times S p(1)} \\
\frac{F_{4(+4)}}{S p(3) \times S p(1)} & \frac{E_{6(+2)}}{S U(6) \times S p(1)} & \frac{E_{7(-5)}}{S O(12) \times S p(1)} & \frac{E_{8(-24)}}{E_{7} \times S p(1)} .
\end{array}
$$

They have the form $G / H \times S p(1)$ with $H \in S p(n)$. The number in brackets for the exceptional groups refers to the choice of a real form of the group. It is equal to the difference between the numbers of noncompact and compact generators for this real form. The compact case corresponds to the compact real form of the numerator.

In the present Letter we construct the Hamiltonians $\mathcal{L}^{+4}$ for all these spaces (some of them have been found in [3]). We find that they have an interesting universal algebraic meaning. To describe it, consider a hamiltonian action of a compact Lie algebra $H$ on a symplectic manifold $M$. Denote generators of $H$ by $\Gamma_{a}$ and functions on $M$ corresponding to $\Gamma_{a}$ by $K_{a}$. Let $g_{a b}$ be an invariant nondegenerate metric on $H$. Then one can form a function $\mathcal{L}=g^{a b} K_{a} K_{b}$, which we will call a Casimir function for its similarity with the Casimir operator. In the case of the quaternionic symmetric space $G / H \times S p(1)$ (which is coordinatized by the set $Q^{+}, q^{+}, u^{-}$) every generator $\Gamma_{A}$ of $G$ maps to a function $K_{A}^{++}\left(Q^{+}, q^{+}, u^{-}\right)$, and the action of $K^{++}$is given by the Poisson brackets (2) with $K^{++}$. The restriction of the Killing-Cartan metric of $G$ to $H$ is nondegenerate. We prove that the potentials for the quaternionic symmetric spaces are given by

$$
\mathcal{L}^{+4}=\frac{(n+2)}{3\left(q^{+i} u_{i}^{-}\right)^{2}} g^{a b} K_{a}^{++} K_{b}^{++},
$$

where $K_{a}^{++}$generate the subgroup $H$ from the denominator and $4 n$ is the space dimension. The quaternionic potentials $\mathcal{L}^{+4}$ are the same in compact and noncompact cases. The only difference is the relative sign of "kinetic" terms for $Q^{+}$and $q^{+}$in the action (四) and in the Poisson brackets (2).

A direct way to prove this statement would be to follow the general procedure [5] which establishes a one-to-one correspondence between the quaternionic spaces and the quaternionic potentials $\mathcal{L}^{+4}$. However, in the case of symmetric spaces the existence of of conserved Killing potentials for the isometry group $G$ turns out to be sufficient to determine the potential $\mathcal{L}^{+4}$. We find $\mathcal{L}^{+4}$ and the set of functions $K_{i j}^{++}, K_{\alpha i}^{++}$and $K_{a}^{++}$ such that all $K^{++}$are conserved in the sense (3), $K_{i j}^{++}$form the Lie algebra of $S p(1)$, $K_{a}^{++}$form the Lie algebra of $H$, and together with $K_{\alpha i}^{++}$they all form the Lie algebra of $G$ - all this with respect to the Poisson brackets (2). The solution to these requirements defines locally a quaternionic manifold with an action of $G$ on it. By the construction, we will see that $H$ and $S p(1)$ are stability subgroups, and the whole space is coordinatized by the coset parameters. Therefore this quaternionic space can be nothing else but the quaternionic symmetric space $G / H \times S p(1)$. 


\section{$2 \quad$ An identity for quaternionic symmetric spaces}

As mentioned in the Introduction every complex simple Lie group $G_{c}$ has two real forms $G$ which contain such a subgroup $H \times S p(1)$ that the coset space $G / H \times S p(1)$ is quaternionic and symmetric. In this section we consider the Jacobi identities which result from the decomposition of $G$ with respect to $H \times S p(1)$ and derive a general identity, which plays a central role in our construction.

We start with writing the Lie algebra of $G$ :

$$
\begin{gathered}
{\left[\Gamma_{\alpha i}, \Gamma_{\beta j}\right]=\epsilon_{i j} t_{\alpha \beta}{ }^{a} \Gamma_{a}+\Omega_{\alpha \beta} \Gamma_{i j},} \\
{\left[\Gamma_{a}, \Gamma_{\alpha i}\right]=t_{a \alpha}{ }^{\beta} \Gamma_{\beta i},} \\
{\left[\Gamma_{a}, \Gamma_{b}\right]=f_{a b}{ }^{c} \Gamma_{c},} \\
{\left[\Gamma_{i j}, \Gamma_{\alpha k}\right]=\epsilon_{i k} \Gamma_{\alpha j}+\epsilon_{j k} \Gamma_{\alpha i}, \quad\left[\Gamma_{i j}, \Gamma_{a}\right]=0,} \\
{\left[\Gamma_{k l}, \Gamma_{i j}\right]=\epsilon_{k i} \Gamma_{l j}+\epsilon_{l i} \Gamma_{k j}+\epsilon_{k j} \Gamma_{l i}+\epsilon_{l j} \Gamma_{k i},}
\end{gathered}
$$

where $\Gamma_{a}, \Gamma_{i j}$ and $\Gamma_{\alpha i}$ are the generators corresponding to the stability subgroups $H$ and $S p(1)$ and the coset space $G / H \times S p(1)$, respectively.

For these commutators to form a Lie algebra a number of Jacobi identities has to be satisfied. The nontrivial identities occur for the following triples : $\left(\Gamma_{\alpha i}, \Gamma_{\beta j}, \Gamma_{\gamma k}\right)$, $\left(\Gamma_{\alpha i}, \Gamma_{\beta j}, \Gamma_{a}\right),\left(\Gamma_{\alpha i}, \Gamma_{a}, \Gamma_{b}\right)$. The first Jacobi identity reads

$$
\begin{gathered}
\epsilon_{i j} t_{\alpha \beta}{ }^{a} t_{a \gamma}{ }^{\sigma} \Gamma_{\sigma k}+\Omega_{\alpha \beta}\left(\epsilon_{i k} \Gamma_{\gamma j}+\epsilon_{j k} \Gamma_{\gamma i}\right)+ \\
\epsilon_{j k} t_{\beta \gamma}{ }^{a} t_{a \alpha}{ }^{\sigma} \Gamma_{\sigma i}+\Omega_{\beta \gamma}\left(\epsilon_{j i} \Gamma_{\alpha k}+\epsilon_{k i} \Gamma_{\alpha j}\right)+ \\
\epsilon_{k i} t_{\gamma \alpha}{ }^{a} t_{a \beta}{ }^{\sigma} \Gamma_{\sigma j}+\Omega_{\gamma \alpha}\left(\epsilon_{k j} \Gamma_{\beta i}+\epsilon_{i j} \Gamma_{\beta k}\right)=0 .
\end{gathered}
$$

Symmetrizing it in $i, j$ we obtain

$$
\Pi_{\gamma \beta \alpha}{ }^{\sigma}-\Pi_{\gamma \alpha \beta}{ }^{\sigma}=\Omega_{\beta \gamma} \delta_{\alpha}^{\sigma}+\Omega_{\gamma \alpha} \delta_{\beta}^{\sigma}-2 \Omega_{\alpha \beta} \delta_{\gamma}^{\sigma},
$$

where

$$
\Pi_{\alpha \beta \gamma}{ }^{\sigma}=t_{\alpha \beta}{ }^{a} t_{a \gamma}{ }^{\sigma} .
$$

Antisymmetrization gives

$$
2 \Pi_{\alpha \beta \gamma}{ }^{\sigma}-\Pi_{\beta \gamma \alpha}{ }^{\sigma}-\Pi_{\gamma \alpha \beta}{ }^{\sigma}=3\left(\Omega_{\beta \gamma} \delta_{\alpha}^{\sigma}-\Omega_{\gamma \alpha} \delta_{\beta}^{\sigma}\right),
$$

which is a consequence of (13), and therefore does not produce new restrictions.

The Jacobi identities for the second and third triples relate the structure constants $f_{a b}{ }^{c}$ with $t_{\alpha \beta}{ }^{a}$ and $t_{a \alpha}{ }^{\beta}$ :

$$
t_{\alpha \beta}^{a} f_{a b}{ }^{c}+t_{\gamma \beta}{ }^{c} t_{b \alpha}{ }^{\gamma}+t_{\gamma \alpha}{ }^{c} t_{b \beta}{ }^{\gamma}=0,
$$

and

$$
t_{c \alpha}{ }^{\gamma} f_{a b}{ }^{c}+t_{a \alpha}{ }^{\beta} t_{b \beta}{ }^{\gamma}-t_{b \alpha}{ }^{\beta} t_{a \beta}{ }^{\gamma}=0 .
$$


The Jacobi identity for the triple $\left(\Gamma_{\alpha i}, \Gamma_{\beta j}, \Gamma_{a}\right)$ gives also

$$
t_{b \alpha}^{\gamma} \Omega_{\gamma \beta}=t_{b \beta}^{\gamma} \Omega_{\gamma \alpha}
$$

which says that $\Gamma_{a}$ generate a subgroup of a symplectic group preserving $\Omega_{\alpha \beta}$.

The Jacobi identity for the structure constants $f_{a b}{ }^{c}$ follows from the Jacobi identities above.

Using (13) and (18) one finds

$$
\Pi_{\gamma \beta \alpha}{ }^{\beta}=(2 n+1) \Omega_{\gamma \alpha} .
$$

We will need some information about the Killing metric for $G$. The involution $\Gamma_{\alpha} \mapsto$ $-\Gamma_{\alpha}, \Gamma_{a} \mapsto \Gamma_{a}$ and $\Gamma_{i j} \mapsto \Gamma_{i j}$, entering the definition of symmetric spaces, leaves the Killing metric $g$ of the group $G$ invariant. Therefore the subspace $\left\{\Gamma_{a}, \Gamma_{i j}\right\}$ is orthogonal to the subspace $\left\{\Gamma_{\alpha}\right\}$. The subspaces $\left\{\Gamma_{a}\right\}$ and $\left\{\Gamma_{i j}\right\}$ are orthogonal as well. In other words the Killing metric is block-diagonal. The components $g_{a b}$ (for the generators of $H$ ) and $g_{\alpha i, \beta j}$ (for the coset generators) of the Killing metric are

$$
\begin{gathered}
g_{a b}=2 t_{a \alpha}{ }^{\beta} t_{b \beta}{ }^{\alpha}+f_{a c}{ }^{d} f_{b d}{ }^{c}, \\
g_{\alpha i, \beta j}=-4(n+2) \epsilon_{i j} \Omega_{\alpha \beta} .
\end{gathered}
$$

Note that the restriction of the Killing metric for $G$ to $H$ is nondegenerate and might not coincide with the Killing metric for $H$ : the subgroup $H$ may contain more than one simple factor, or a $U(1)$ factor.

With the help of eq-ns $(20)$ and (21) the antisymmetry of the structure constants implies

$$
g_{a b} t_{\alpha \beta}^{b}=4(n+2) \Omega_{\beta \gamma} t_{a \alpha}^{\gamma}
$$

Define a quantity

$$
\Pi_{\alpha \beta \gamma \delta}=\Omega_{\delta \sigma} \Pi_{\alpha \beta \gamma}{ }^{\sigma} .
$$

It is symmetric in $\alpha, \beta$ and in $\gamma, \delta$. Moreover, (22) implies that it is invariant under $\alpha \beta \leftrightarrow \gamma \delta$ as well. Therefore the totally symmetric part $S_{\alpha \beta \gamma \delta}$ of $\Pi_{\alpha \beta \gamma \delta}$ is $S_{\alpha \beta \gamma \delta}=\left(\Pi_{\alpha \beta \gamma \delta}+\right.$ $\left.\Pi_{\alpha \gamma \beta \delta}+\Pi_{\alpha \delta \gamma \beta}\right) / 3$. It follows from (13) that $S$ can be written in the form

$$
S_{\alpha \beta \gamma \delta}=\Pi_{\alpha \beta \gamma \delta}+\Omega_{\alpha \gamma} \Omega_{\beta \delta}+\Omega_{\alpha \delta} \Omega_{\beta \gamma} .
$$

Now we are going to derive an identity for the tensor $S_{\alpha \beta \gamma \delta}$. To this end we multiply (17) by $t_{\mu \nu}{ }^{a} t_{\sigma \tau}{ }^{b}$ and use (16) to eliminate the structure constants $f_{a b}{ }^{c}$ :

$$
\Pi_{\rho \mu \alpha}^{\gamma} \Pi_{\sigma \tau \nu}^{\rho}+\Pi_{\rho \nu \alpha}^{\gamma} \Pi_{\sigma \tau \mu}^{\rho}+\Pi_{\mu \nu \rho}^{\gamma} \Pi_{\sigma \tau \alpha}^{\rho}-\Pi_{\sigma \tau \rho}^{\gamma} \Pi_{\mu \nu \alpha}^{\rho}=0 .
$$

This identity has the following algebraic meaning. One can find the structure constants $f_{a b}{ }^{c}$ using either of eq-ns (16) or (17). Therefore there is a compatibility condition ensuring that we obtain the same $f_{a b}{ }^{c}$, which is exactly (25).

Symmetrizing (25) in lower indices $\mu, \nu, \alpha, \sigma$ and $\tau$ and expressing $\Pi$ in terms of completely symmetric tensor $S(24)$ we obtain the identity

$$
S_{\rho(\mu \alpha}^{\gamma} S_{\sigma \tau \nu)}{ }^{\rho}-\delta_{(\mu}^{\gamma} S_{\alpha \sigma \tau \nu)}=0
$$

It holds universally for all symmetric spaces (5). This identity plays fundamental role in the construction of the quaternionic potentials for these spaces in the next Section. 


\section{Killing potentials and the Hamiltonian}

Now we use the results of the previous section to find the Hamiltonians and Killing potentials for the quaternionic symmetric spaces. First of all we remark that the $S p(1)$ invariance requires the indices $i$ of $u_{i}^{-}$or $q^{+i}$ in $\mathcal{L}^{+4}\left(Q^{+}, q^{+}, u^{-}\right)$to be contracted invariantly. The only nonvanishing combination satisfying this condition is $\left(q^{+} u^{-}\right)=q^{+i} u_{i}^{-}$. Therefore $\mathcal{L}^{+4}$ depends only on $Q^{+}$and $\left(q^{+} u^{-}\right)$,

$$
\mathcal{L}^{+4}=\mathcal{L}^{+4}\left(Q^{+},\left(q^{+} u^{-}\right)\right) .
$$

Since $\mathcal{L}^{+4}$ has a $U(1)$-charge 4 , the form (27) implies that $\mathcal{L}^{+4}$ is homogeneous of degree 4 in $Q^{+}$. Moreover, since $Q^{+}$is a remnant of coset coordinates corresponding to coset generators, the point $Q^{+}=0$ must be nonsingular. Therefore $\mathcal{L}^{+4}$ must be proportional to a fourth order polynomial in $Q^{+}$. Finally, since $\mathcal{L}^{+4}$ is homogeneous of degree 2 in $Q^{+}, q^{+}$, the above arguments restrict $\mathcal{L}^{+4}$ to be

$$
\mathcal{L}^{+4}=\frac{P^{+4}\left(Q^{+}\right)}{\left(q^{+} u^{-}\right)^{2}}
$$

with a fourth order polynomial $P^{+4}$. We are going to prove that

$$
P^{+4}\left(Q^{+}\right)=\xi S_{\alpha \beta \gamma \delta} Q^{+\alpha} Q^{+\beta} Q^{+\gamma} Q^{+\delta}
$$

with a constant $\xi$ to be defined later.

As explained in the Introduction, our proof consists of giving explicit expressions for the Killing potentials. We take

$$
\begin{aligned}
S p(1): & K_{i j}^{++}=2\left(q_{i}^{+} q_{j}^{+}-u_{i}^{-} u_{j}^{-} \mathcal{L}^{+4}\right), \\
H: & K_{a}^{++}=t_{a \alpha \beta} Q^{+\alpha} Q^{+\beta} \\
G / H \times S p(1): & K_{i \alpha}^{++}=2 q_{i}^{+} Q_{\alpha}^{+}-u_{i}^{-}\left(q^{+} u^{-}\right) \partial_{\alpha}^{-} \mathcal{L}^{+4},
\end{aligned}
$$

where $\partial_{\alpha}^{-}$is the partial derivative with respect to $Q^{+\alpha}$, and $t_{a \alpha \beta}=\Omega_{\beta \gamma} t_{a \alpha}{ }^{\gamma}$. It is straightforward to show that the $S p(1)$ potential $K_{i j}^{++}$is conserved for an arbitrary polynomial $P^{+4}\left(Q^{+}\right)$. The conservation law for $K_{a}^{++}$is satisfied for $P^{+4}$ given by (29) but still does not fix $\xi$. (With the choice (31) the fourth order polynomial $P^{+4}$ (29) is proportional to $g^{a b} K_{a}^{++} K_{b}^{++}$. Therefore it is similar to the second order Casimir operator for the group $H$ and this makes the conservation of $K_{a}^{++}$obvious.)

A Killing potential $K^{++}$induces the transformation

$$
\delta Q^{+\alpha} \sim\left\{Q^{+\alpha}, K^{++}\right\}
$$

on the coordinates $Q^{+}$. The origin $Q^{+\alpha}=0$ is stable under the transformations induced by the Killing potentials $K_{i j}^{++}$and $K_{a}^{++}$but not $K_{i \alpha}^{++}$which shows that the stability subgroup is precisely $H \times S p(1)$.

Finally, a direct calculation shows that the conservation law for the coset potential is reduced to the equation

$$
Q_{\alpha}^{+} P^{+4}-\frac{1}{2}\left\{\partial_{\alpha}^{-} P^{+4}, P^{+4}\right\}^{--}=0
$$


It imposes a nontrivial restriction on the structure constants $t_{a \alpha}{ }^{\beta}$ and $t_{\alpha \beta}{ }^{a}$. Substituting (29) into (34) we obtain an algebraic equation for $S$,

$$
\left[\Omega_{\alpha \mu} S_{\nu \rho \sigma \gamma}-12 \xi S_{\alpha \mu \nu \tau} S_{\rho \sigma \gamma}{ }^{\tau}\right] Q^{+\mu} Q^{+\nu} Q^{+\rho} Q^{+\sigma} Q^{+\gamma}=0
$$

Comparing this with eq. (26) we find $\xi=1 / 12$. Then eq-ns $(28),(29)$ together with (22) fix the Hamiltonian to the form (6). One can similarly express $P^{+4}$ in terms of the coset Killing potentials, or the $S p(1)$ Killing potentials:

$$
P^{+4}=-\frac{1}{16} \epsilon^{i j} \Omega^{\alpha \beta} K_{i \alpha}^{++} K_{j \beta}^{++}=-\frac{1}{8} K^{++i j} K_{i j}^{++} .
$$

It is straightforward to check that the Killing potentials given in (30), (31) and (32) generate precisely the algebra (1)-(11).

In summary, we have found the harmonic potentials for the quaternionic symmetric spaces. They turned out to be simple fourth order polynomials in $Q^{+}$similar to the quadratic Casimir operator of the subgroup $H$. It seems plausible that a more general class of homogeneous nonsymmetric quaternionic spaces of Alekseevskii [9] can be described in the same way.

Acknowledgements. We are grateful to E. Ivanov and V. Ogievetsky for their interest and valuable discussions. A.G. acknowledges support by the U.S. National Science Foundation, grant PHY-90096198. O.O. would like to thank the Particle Physics Group of the Johns Hopkins University for hospitality during the course of the work.

\section{References}

[1] T. Eguchi, P.B. Gilkey and A.J. Hanson, Phys. Reports 66 (1980) 213.

[2] J. Bagger and E. Witten, Nucl. Phys. B 222 (1983) 1.

[3] J.A. Bagger, A.S. Galperin, E.A. Ivanov and V.I. Ogievetsky, Nucl. Phys. B 303 (1988) 522.

[4] A. Galperin, E. Ivanov, S. Kalitzin, V. Ogievetsky and E. Sokatchev, Class. Quantum Grav. 1 (1984) 469.

[5] A. Galperin, E. Ivanov and O. Ogievetsky, Harmonic Space Description of Quaternionic Manifolds, preprint JHU-TIPAC-920023, MPI-Ph/92-85, ENSLAPPL-405/992.

[6] B. Zumino, Phys. Lett. B 87 (1979) 203.

[7] A. Galperin and V. Ogievetsky, Class. Quantum Grav. 8 (1991), 1757.

[8] J. Wolf, J. Math. Mech. 14 (1965) 1033.

[9] D.A. Alekseevskii, Math. USSR Izvestija 9 (1975) 297. 